\title{
Possible Contribution of PTSD to Altered Cortisol Activity in Young Adult Obese African-American Women
}

\author{
Teletia R. Taylor • Kendra Van Kirk • Denia Tapscott • \\ Monet Bernard • Juliana Llano • Thomas A. Mellman
}

Received: 24 June 2014 / Revised: 2 September 2014 / Accepted: 24 October 2014 / Published online: 6 December 2014

(C) W. Montague Cobb-NMA Health Institute 2014

\begin{abstract}
Objective African-Americans have been found to experience increased rates of post-traumatic stress disorder (PTSD), obesity, and flatter diurnal cortisol slopes compared to other demographic groups. Further exploration, however, is needed to understand how PTSD impacts diurnal cortisol activity in obese African-American women. The purpose of the current study is to examine the relationship between salivary cortisol levels and PTSD in a sample of obese young adult AfricanAmerican women and to examine how depression and insomnia influence the relationship.

Methods Thirty-four young adult African-American women (mean age $=24.0$ years; mean $\mathrm{BMI}=37.4 \mathrm{~kg} / \mathrm{m}^{2}, 6 / 34$ of the sample had a score of 40 or above on the PTSD Checklist (PCL) representing clinically significant PTSD) filled out questionnaires assessing PTSD, lifetime exposure to traumatic events, insomnia severity, and depression. A home-based assessment of salivary cortisol was provided upon awakening at $30 \mathrm{~min}$ and $1,3,6$, and $12 \mathrm{~h}$.

Results There was a significant interaction between PTSD status and diurnal cortisol activity $(p<0.04)$. There were trends for higher cortisol levels at awakening $(p<0.051)$ and
\end{abstract}

\footnotetext{
T. R. Taylor $(\varangle) \cdot$ K. Van Kirk $\cdot$ J. Llano

College of Medicine, Howard University, 2041 Gerogia Avenue,

NW, Washington, DC 20060, USA

e-mail: t_r_taylor@howard.edu

D. Tapscott

Department of Internal Medicine, Howard University, Washington, DC, USA

\section{Bernard • T. A. Mellman}

Department of Psychiatry and Behavioral Sciences, Howard

University, Washington, DC, USA

T. A. Mellman

Center for Clinical and Translational Research, Howard University,

Washington, DC, USA
}

30 min post-awakening $(p<0.059)$ with PTSD. The significance of the interaction between PTSD and cortisol was attenuated by co-varying for depression and insomnia $(p>0.05)$.

Conclusion PTSD, influenced by depression and insomnia symptoms, has an impact on diurnal cortisol activity in obese young adult African-American women.

Keywords Post-traumatic stress disorder .

African-Americans $\cdot$ Obesity $\cdot$ Cortisol

\section{Introduction}

Post-traumatic stress disorder (PTSD) is a prevalent condition and confers elevated risk for several mental and physical health conditions $[1,2]$. African-American women have elevated rates of PTSD as well as associated medical conditions such as obesity [3]. Roberts et al. [4] documented higher rates of lifetime prevalence of PTSD among African-Americans (8.7\%) compared with non-Latino whites $(7.4 \%)$ or Asians (4.0\%). Breslau et al. [5] found that women were more than two times as likely as men (30.2 vs. $13 \%$, respectively) to develop PTSD following a traumatic event.

PTSD has been associated with obesity in AfricanAmerican women [6]. For example, Mitchell et al. [6] investigated the comorbidity of lifetime PTSD and overweight/ obesity in a population-based African-American urban sample. Data were obtained from 463 African-American male and female participants of the Detroit Neighborhood Health Study. In sex-stratified models, after controlling for demographic variables, PTSD was associated with obesity only among women.

One of the mechanisms by which PTSD can affect physical health, including obesity, is through dysregulated hypothalamic-pituitary-adrenal (HPA) axis functioning [7]. 
The HPA axis is an important contributor to the human stress response by releasing corticotropin-releasing factor (CRF) from the hypothalamus, which in turn stimulates the secretion of adrenocorticotropin-releasing hormone (ACTH) from the pituitary gland. ACTH, in turn, stimulates the release of cortisol from the adrenal glands and serves as part of a feedback loop to regulate HPA axis activity [7].

Research evaluating HPA axis functioning in PTSD has yielded mixed findings. The most comprehensive studies have assessed male combat veterans and have found low cortisol levels related to increased feedback inhibition in veterans with PTSD compared with normal controls and combat controls [8]. It is not clear to what degree these findings generalize to populations other than male combat veterans with chronic and severe PTSD. In fact, a number of studies of cortisol and PTSD using non-veteran subjects recruited from clinics and the community have failed to confirm low cortisol $[9,10]$ and some studies demonstrated increased cortisol with PTSD [11]. African-Americans have been found to have flatter diurnal cortisol slopes compared to other demographic groups [12] and, as previously noted, experience increased rates of PTSD [4].

In exploring the association between cortisol and PTSD, it is important to examine other factors contributing to the relationship. For example, depression and sleep disturbance occur commonly with PTSD $[13,14]$ and both depression and sleep disturbances impact cortisol activity $[15,16]$. Moreover, sleep disturbance and depression are associated with obesity in African-American women. For example, in a convenience sample of 69 obese young adult African-American women, it was reported that BMI was associated with sleep latency [17]. Also, in an analysis of the National Survey of American Life (NSAL), a household mental health survey of adult Black Americans [18], it was reported that for Black Caribbean women, $\mathrm{BMI} \geq 30 \mathrm{~kg} / \mathrm{m}^{2}$ and $\mathrm{BMI} \geq 35 \mathrm{~kg} / \mathrm{m}^{2}$ were associated with higher odds of 12 months major depressive disorder (MDD). Although non-significant, among African-American women, $\mathrm{BMI} \geq 30, \mathrm{BMI} \geq 35$, and $\mathrm{BMI} \geq 40 \mathrm{~kg} / \mathrm{m}^{2}$ were all associated with higher odds of 12 months MDD.

Studies linking cortisol with obesity have shown mixed results. Higher waist-to-hip ratios have been associated with lower diurnal cortisol variability [19, 20]. While cortisol awakening response has been positively associated with waist-to-hip ratio and waist circumference [21, 22], others have found an inverse association between awakening and morning cortisols and body mass index (BMI) [23, 24]. Further research is needed to elucidate these relationships. The purpose of the current study is to examine the relationship between salivary cortisol levels and PTSD in a sample of young adult, obese, African-American women. We also sought to examine how depression and insomnia influence the relationship.

\section{Methods}

Participants

Recruitment for the study occurred between April 2011 and May 2012. African-American females aged 18 to 35 were recruited via flyers advertising a weight loss intervention study. The flyers were posted around Howard University Hospital, Howard University College of Medicine, and the undergraduate campus in addition to recreation centers, churches, supermarkets, and childcare centers in the nearby community. Exclusion criteria included age $<18$ or $>35$, body mass index $(\mathrm{BMI})<30$, currently on a formal diet, current use of weight loss medications, current alcohol or illicit drug abuse or dependence, use of medications for any psychiatric disorder, diabetes, hypertension, hypercholesterolemia, or use of any other cardiac medications. All participants signed an informed consent form that was approved by the University Institutional Review Board after procedures had been explained and their questions had been answered.

\section{Screening and Evaluation}

Participants were screened with questions regarding height and weight, health status, previous/current use of weight loss interventions, and substance abuse/addiction. For screening purposes, BMI was calculated with an online BMI calculator tool using the height and weight verbally provided by participants (actual BMI was determined during the baseline visit).

\section{Procedures}

Each participant completed a baseline visit in which height, weight, waist circumference, and BMI were measured at Howard University Hospital's Clinical Research Unit (CRU). The following questionnaires were administered to participants: The PTSD Checklist (PCL) is a well-validated screening tool for measuring current (experienced within the past month) symptoms of PTSD [25]. A score of 40 or higher indicates clinically relevant PTSD. It was administered in conjunction with the Life Events Checklist (LEC), an inventory of lifetime exposure to traumatic events [26]. Both scales have been used to measure PTSD and exposure to traumatic events in African-American samples [27, 28]. The Patient Health Questionnaire (PHQ-9), a nine-item self-report measure of depression, has been validated in ethnically diverse populations including African-Americans [29] and is widely used in primary care settings [30]. PHQ-9 scores range from 0 to 27 , and a score of 10 and above indicates depression of moderate or greater severity. The Insomnia Severity Index (ISI) is a selfreport measure that assesses subjective severity of insomnia over a 2-week period [31] and has been used to measure insomnia in African-American female samples [32, 33]. ISI 
scores range from 0 to 28 and a score above 10 represents clinically significant insomnia symptoms.

A home-based assessment of salivary cortisol was conducted, and participants were instructed to provide saliva in a collection tube upon awakening at $30 \mathrm{~min}$ and 1, 3, 6, and $12 \mathrm{~h}$. Sample tubes were returned to the CRU for analysis and storage.

\section{Statistical Analysis}

Means, standard deviations, and percentages were calculated to determine group characteristics. Diurnal cortisol responses by PTSD group membership were analyzed with repeated measures analysis of variance (ANOVA) with sample time (awakening, $30 \mathrm{~min}$ and 1, 3, 6, and $12 \mathrm{~h}$ ) as the within-subject factor and PTSD status (equal to or above 40 on the PCL scale, PTSD positive) as the between-subject factor. A separate repeated measures ANOVA was conducted controlling for Insomnia Severity Index scores and PHQ-9 scores.

\section{Results}

All of the 34 participants were black, 29 self-identified as African-American, and 5 as West Indian/Caribbean. The mean (standard deviation) (SD) age of the group was 24.0 (4.6) years (range $=18-32$ years). The mean (SD) BMI of the group was $37.4(6.3) \mathrm{kg} / \mathrm{m}^{2}$. Mean (SD) PCL scores were 30.2 (11.8), and $18 \%(6 / 34)$ of the sample had a score of 40 or above representing clinically significant PTSD. Based on the Life Event Checklist, the six participants scoring 40 or above on the PCL reported traumatic events that included losing children, being molested as a child, and the traumatic death of a caretaker at age 13. Mean (SD) PHQ-9 scores were 6.0 (5.9) with $18 \%(6 / 34)$ of the sample meeting the criteria for moderate and greater severity of depression. Mean (SD) ISI scores were 8.1 (5.8) with $41 \%$ (14/34) of the sample meeting the threshold for clinically significant insomnia symptoms.

There was a significant interaction between PTSD status and diurnal cortisol activity $(p<0.040)$. There were trends for higher cortisol levels at awakening $(p<0.051)$ and $30 \mathrm{~min}$ post-awakening $(p<0.059)$ with PTSD (see Fig. 1$)$. The significance of the interaction between PTSD and cortisol was attenuated by co-varying for depression and insomnia $(p>0.05)$ (see Fig. 2).

\section{Discussion}

Our findings provide preliminary evidence for a relationship between diurnal cortisol and PTSD in young adult, obese, African-American women with trends for higher cortisol activity soon after awakening in participants with PTSD. Johnson et al. [34] found an association of PTSD severity with significantly greater cortisol output the first hour after awakening. As reviewed in the introduction, research evaluating HPA axis functioning in PTSD has yielded mixed results. The most comprehensive studies have assessed male combat veterans and have found low cortisol levels in veterans with PTSD [8]. The fact that we found disparate results from male veteran studies confirms that our sample may differ from male veterans in important ways. It also suggests that more work should be done examining the biology of PTSD in community samples such as ours.

Our study also showed that the association between diurnal cortisol and PTSD status was partially accounted for by insomnia and depression. These findings are not surprising given that sleep disturbance such as trouble falling and staying asleep (insomnia) are common symptoms of PTSD [14], and sleep impairments are known to be associated with alterations in the HPA axis [15]. In addition, research documents a cooccurrence of depression and PTSD [13] as well as elevations in salivary cortisol in patients with MDD [16].

Limitations of the current study include its small sample size and the low number of PTSD respondents. Another limitation is the study only addressed women. As a result, we were not able to determine gender differences pertaining to the effect of PTSD on cortisol. Laudenslager et al. [35] explored diurnal rhythms in salivary cortisol and PTSD in a sample of American-Indian men and women. When stratified by gender, women with a lifetime diagnosis of PTSD had significantly higher mean cortisol levels throughout the day than women without PTSD, but there was no significant association between PTSD and cortisol levels in men. Adding young adult African-American men to a future study would allow for male-female comparisons with respect to biomarkers and PTSD.

Finally, the parent study from which this sample was derived was composed of obese women. Therefore, the generalizability of the findings is limited to this particular anthropometric group. A future investigation could examine similar research questions by comparing a group of non-obese African-American women with and without PTSD to a group of obese African-American women with and without PTSD. One could explore if PTSD is more prevalent in an obese group. This association could be related to disruptions of inhibitory control in PTSD largely mediated by the prefrontal cortex [36]. The capacity of inhibitory control is critical to suppressing food intake in a modern environment characterized by frequent exposure to highly palatable food [37]. Based on these findings, it appears plausible that the neurobiological disruption of inhibitory control associated with PTSD may make one vulnerable to overeating and obesity. Even though evidence indicates significant group differences between African-American women and Caucasian women in their 
Fig. 1 Time point 1 represents awakening, 2 equals $30 \mathrm{~min}, 3$ equals 1 h, 4 equals 3 h, 5 equals $6 \mathrm{~h}$, and 6 equals $12 \mathrm{~h}$

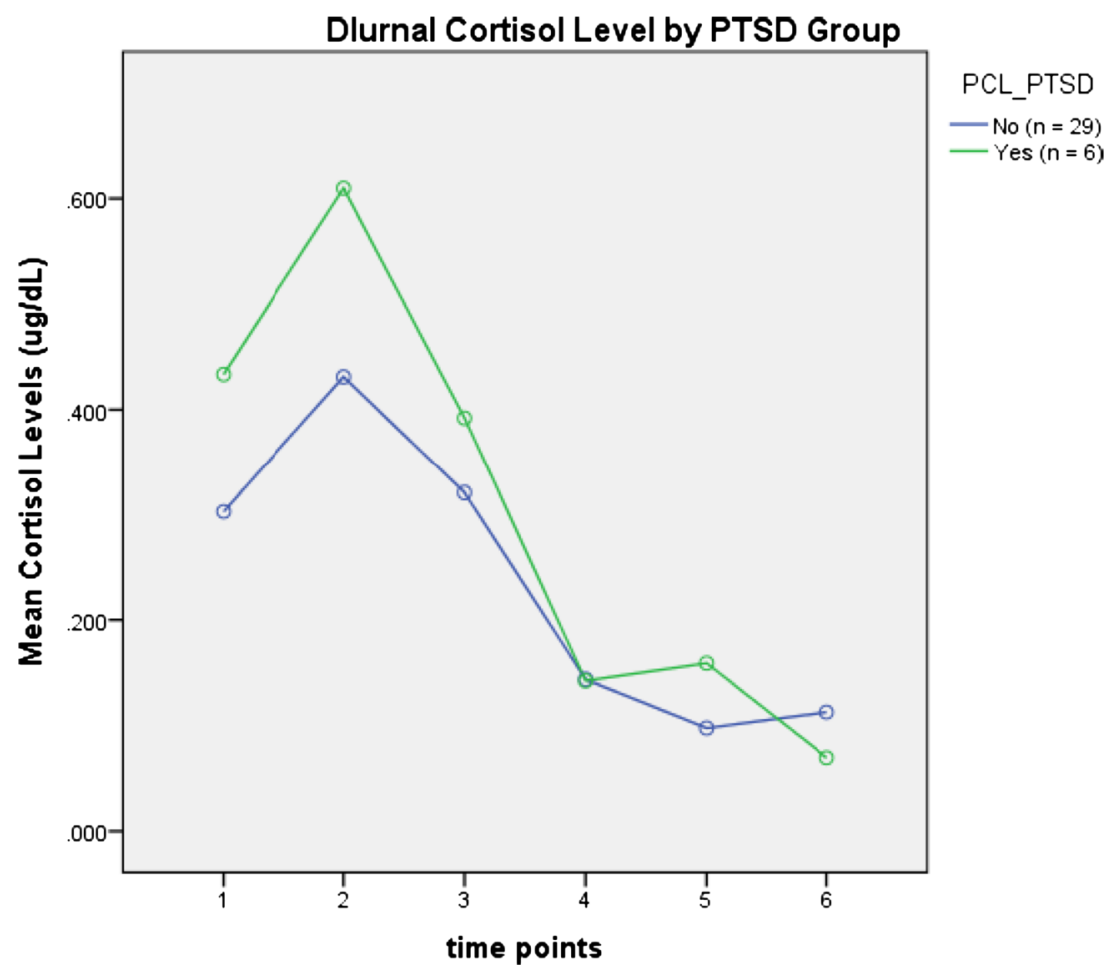

perceptions of their overweight and obese status [38], conducting a comparison among African-American women with and without PTSD and obesity would further elucidate our understanding of the PTSD/cortisol relationship within this ethnic group as well as factors mediating the relationship.

Our findings linking PTSD with cortisol justify the importance of the accurate diagnosis and treatment of PTSD in populations of young adult African-American women. As previously mentioned, the health consequences of PTSD are substantial and can include secondary mental disorders [39], substance use disorders [40], and a range of physical conditions [41]. Effective intervention strategies include cognitive behavioral therapy that has proven to be a useful intervention technique for the treatment of PTSD in a variety of samples including African-American women [42] .

In summary, African-Americans in general have been found to experience increased rates of PTSD [4] and flatter diurnal cortisol slopes compared to other demographic groups [12]. Findings of this study provide further evidence that PTSD, influenced by depression and insomnia symptoms,
Fig. 2 Time point 1 represents awakening, 2 equals $30 \mathrm{~min}, 3$ equals $1 \mathrm{~h}, 4$ equals $3 \mathrm{~h}, 5$ equals $6 \mathrm{~h}$, and 6 equals $12 \mathrm{~h}$
Diurnal Cortisol levels by PTSD Group Controlling for Depression and Insomnia

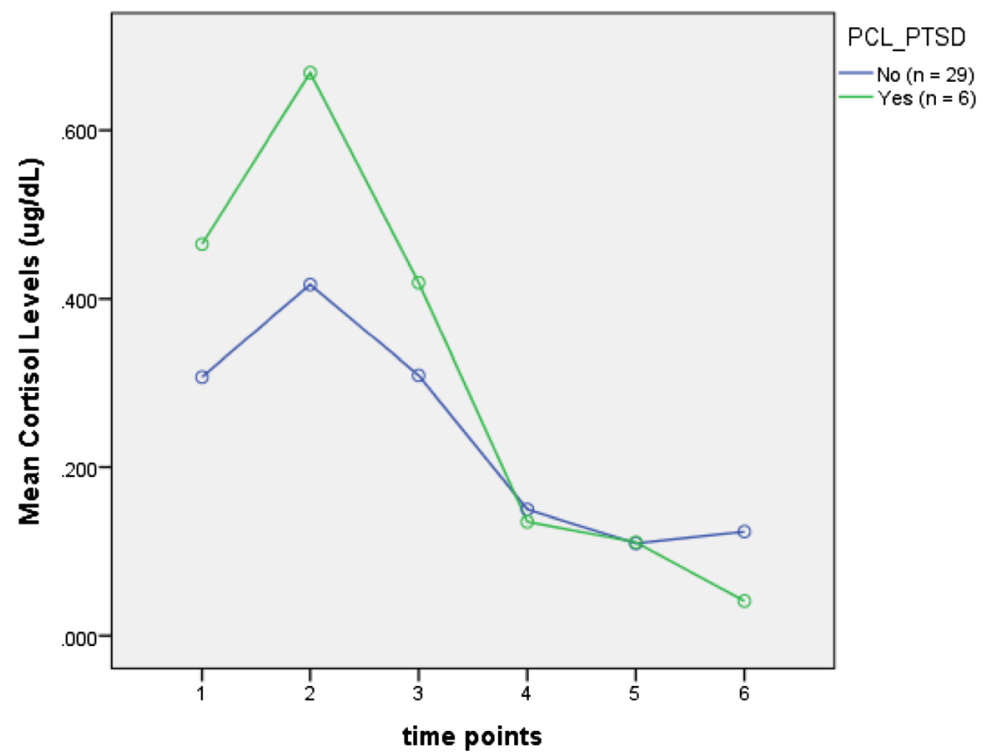


has an impact on diurnal cortisol activity in young adult, obese African-American women that should be confirmed and extended by future research.

Source of Funding This work was sponsored by the collaborative studies grant of the Georgetown-Howard Universities Center for Clinical and Translational Science. UL1TR000101 previously UL1RR031975 from the National Center for Advancing Translational Sciences (NCATS).

Conflict of Interest Teletia R. Taylor, Ph.D., Kendra Van Kirk, BA, Denia Tapscott, M.D., Monet Bernard, BS, Juliana Llano, BS, and Thomas A. Mellman, M.D. declare that they have no conflict of interest.

Informed Consent Statement All procedures followed were in accordance with the ethical standards of the responsible committee on human experimentation (institutional and national) and with the Helsinki Declaration of 1975, as revised in 2000 (5). Informed consent was obtained from all patients for being included in the study.

\section{References}

1. Weiss T, Skelton K, Phifer J, Jovanovic T, Gillespie CF, Smith A, et al. Posttraumatic stress disorder is a risk factor for metabolic syndrome in an impoverished urban population. Gen Hosp Psychiatry. 2011;33:135-42.

2. Nixon RD, Resick PA, Nishith P. An exploration of comorbid depression among female victims of intimate partner violence with posttraumatic stress disorder. J Affect Disord. 2004;82:315-20.

3. Weinsier RL, Hunter GR, Zuckerman PA, Redden DT, Darnell BE, Larson DE, et al. Energy expenditure and free-living physical activity in black and white women: comparison before and after weight loss. Am J Clin Nutr. 2000;71:1138-46.

4. Roberts AL, Gilman SE, Breslau J, Breslau N, Koenen KC. Race/ethnic differences in exposure to traumatic events, development of post-traumatic stress disorder, and treatment-seeking for posttraumatic stress disorder in the United States. Psychol Med. 2011;41:71-83.

5. Breslau N, Davis GC, Andreski P, Peterson EL, Schultz LR. Sex differences in posttraumatic stress disorder. Arch Gen Psychiatry. 1997;54:1044-8.

6. Mitchell KS, Aiello AE, Galea S, Uddin M, Wildman D, Koenen KC. PTSD and obesity in the Detroit neighborhood health study. Gen Hosp Psychiatry. 2013;35:671-3.

7. Stoppelbein L, Greening L, Fite P. The role of cortisol in PTSD among women exposed to a trauma-related stressor. J Anxiety Disord. 2012;26:352-8.

8. Yehuda R. Current status of cortisol findings in post-traumatic stress disorder. Psychiatr Clin North Am. 2002;25:341-68. vii.

9. Lemieux AM, Coe CL. Abuse-related posttraumatic stress disorder: evidence for chronic neuroendocrine activation in women. Psychosom Med. 1995;57:105-15.

10. Rasmusson AM, Lipschitz DS, Wang S, Hu S, Vojvoda D, Bremner $\mathrm{JD}$, et al. Increased pituitary and adrenal reactivity in premenopausal women with posttraumatic stress disorder. Biol Psychiatry. 2001;50: 965-77.

11. Maes M, Lin A, Bonaccorso S, van Hunsel F, Van Gastel A, Delmeire $\mathrm{L}$, et al. Increased 24-hour urinary cortisol excretion in patients with post-traumatic stress disorder and patients with major depression, but not in patients with fibromyalgia. Acta Psychiatr Scand. 1998;98: 328-35.
12. Cohen S, Schwartz JE, Epel E, Kirschbaum C, Sidney S, Seeman T. Socioeconomic status, race, and diurnal cortisol decline in the Coronary Artery Risk Development in Young Adults (CARDIA) study. Psychosom Med. 2006;68:41-50.

13. Rytwinski NK, Scur MD, Feeny NC, Youngstrom EA. The cooccurrence of major depressive disorder among individuals with posttraumatic stress disorder: a meta-analysis. J Trauma Stress. 2013;26:299-309.

14. Mellman TA, Nolan B, Hebding J, Kulick-Bell R, Dominguez R. A polysomnographic comparison of veterans with combat-related PTSD, depressed men, and non-ill controls. Sleep. 1997;20:46-51.

15. Balbo M, Leproult R, Van Cauter E. Impact of sleep and its disturbances on hypothalamo-pituitary-adrenal axis activity. Int $\mathrm{J}$ Endocrinol. 2010;2010:759234.

16. Jarcho MR, Slavich GM, Tylova-Stein H, Wolkowitz OM, Burke HM. Dysregulated diurnal cortisol pattern is associated with glucocorticoid resistance in women with major depressive disorder. Biol Psychol. 2013;93:150-8.

17. Jarosz PA, Davis JE, Yarandi HN, Farkas R, Feingold E, Shippings $\mathrm{SH}$, et al. Obesity in urban women: associations with sleep and sleepiness, fatigue and activity. Womens Health Issues. 2014;24: e447-54.

18. Assari S. Association between obesity and depression among American Blacks: role of ethnicity and gender. J Racial Ethn Health Disparities. 2014;1:36- 44.

19. Rosmond R, Dallman MF, Bjorntorp P. Stress-related cortisol secretion in men: relationships with abdominal obesity and endocrine, metabolic and hemodynamic abnormalities. J Clin Endocrinol Metab. 1998;83:1853-9.

20. Bjorntorp P, Holm G, Rosmond R. Hypothalamic arousal, insulin resistance and type 2 diabetes mellitus. Diabet Med. 1999;16:37383.

21. Therrien F, Drapeau V, Lalonde J, Lupien SJ, Beaulieu S, Tremblay A, et al. Awakening cortisol response in lean, obese, and reduced obese individuals: effect of gender and fat distribution. Obesity (Silver Spring). 2007;15:377-85.

22. Wallerius S, Rosmond R, Ljung T, Holm G, Bjorntorp P. Rise in morning saliva cortisol is associated with abdominal obesity in men: a preliminary report. J Endocrinol Invest. 2003;26:616-9.

23. Boyle SH, Surwit RS, Georgiades A, Brummett BH, Helms MJ, Williams RB, et al. Depressive symptoms, race, and glucose concentrations: the role of cortisol as mediator. Diabetes Care. 2007;30: 2484-8.

24. Kumari M, Chandola T, Brunner E, Kivimaki M. A nonlinear relationship of generalized and central obesity with diurnal cortisol secretion in the Whitehall II study. J Clin Endocrinol Metab. 2010;95:4415-23.

25. Blanchard EB, Jones-Alexander J, Buckley TC, Forneris CA. Psychometric properties of the PTSD Checklist (PCL). Behav Res Ther. 1996;34:669-73.

26. Gray MJ, Litz BT, Hsu JL, Lombardo TW. The psychometric properties of the Life Events Checklist. Assessment. 2004;11:330-41.

27. Alim TN, Graves E, Mellman TA, Aigbogun N, Gray E, Lawson W, et al. Trauma exposure, posttraumatic stress disorder and depression in an African-American primary care population. J Natl Med Assoc. 2006;98:1630-6.

28. Goldmann E, Aiello A, Uddin M, Delva J, Koenen K, Gant LM, et al. Pervasive exposure to violence and posttraumatic stress disorder in a predominantly African American Urban Community: the Detroit Neighborhood Health Study. J Trauma Stress. 2011;24:747-51.

29. Huang FY, Chung H, Kroenke K, Delucchi KL, Spitzer RL. Using the Patient Health Questionnaire-9 to measure depression among racially and ethnically diverse primary care patients. J Gen Intern Med. 2006;21:547-52.

30. Spitzer RL, Kroenke K, Williams JB. Validation and utility of a selfreport version of PRIME-MD: the PHQ primary care study. Primary 
care evaluation of mental disorders. Patient Health Questionnaire. JAMA. 1999;282:1737-44.

31. Bastien CH, Vallieres A, Morin CM. Validation of the Insomnia Severity Index as an outcome measure for insomnia research. Sleep Med. 2001;2:297-307.

32. Ensrud KE, Joffe H, Guthrie KA, Larson JC, Reed SD, Newton KM, et al. Effect of escitalopram on insomnia symptoms and subjective sleep quality in healthy perimenopausal and postmenopausal women with hot flashes: a randomized controlled trial. Menopause. 2012;19: 848-55.

33. Taylor TR, Huntley ED, Makambi K, Sween J, Adams-Campbell LL, Frederick W, Mellman TA. Understanding sleep disturbances in African-American breast cancer survivors: a pilot study. Psychooncology. 2011.

34. Johnson DM, Delahanty DL, Pinna K. The cortisol awakening response as a function of PTSD severity and abuse chronicity in sheltered battered women. J Anxiety Disord. 2008;22:793-800.

35. Laudenslager ML, Noonan C, Jacobsen C, Goldberg J, Buchwald D, Bremner JD, et al. Salivary cortisol among American Indians with and without posttraumatic stress disorder (PTSD): gender and alcohol influences. Brain Behav Immun. 2009;23:658-62.
36. Lagarde G, Doyon J, Brunet A. Memory and executive dysfunctions associated with acute posttraumatic stress disorder. Psychiatry Res. 2010;177:144-9.

37. Appelhans BM. Neurobehavioral inhibition of reward-driven feeding: implications for dieting and obesity. Obesity (Silver Spring). 2009; 17:640-7.

38. Ard JD, Greene LE, Malpede CZ, Jefferson WK. Association between body image disparity and culturally specific factors that affect weight in Black and White women. Ethn Dis. 2007;17:S2,34-9.

39. Breslau N. Epidemiologic studies of trauma, posttraumatic stress disorder, and other psychiatric disorders. Can J Psychiatry. 2002;47: 923-9.

40. Breslau N, Davis GC, Schultz LR. Posttraumatic stress disorder and the incidence of nicotine, alcohol, and other drug disorders in persons who have experienced trauma. Arch Gen Psychiatry. 2003;60:289-94.

41. Mellman TA, Brown DD, Jenifer ES, Hipolito MM, Randall OS. Posttraumatic stress disorder and nocturnal blood pressure dipping in young adult African Americans. Psychosom Med. 2009;71:627-30.

42. Zoellner LA, Feeny NC, Fitzgibbons LA, Foa EB. Response of African American and Caucasian women to cognitive behavior therapy for PTSD. Behav Ther. 1999;30:581-95. 